\title{
Construyendo Europolis a partir de la cooperación local "informal” en los espacios transfronterizos de Europa: el caso de la RIET ${ }^{*}$
}

\author{
Luis DOMÍNGUEZ CASTRO \\ Departamento de Historia, Arte y Geografía \\ Universidade de Vigo \\ dominguez@uvigo.es
}

Enrique José VARELA ÁlVAREZ

Departamento de Sociología, Ciencia Política y de la Administración y Filosofía

Universidade de Vigo

evalvarez@uvigo.es

Recibido: 23-02-2015

Aceptado: 17-10-2015

\begin{abstract}
RESUMEN
Transcurridos veinticinco años del inicio de la cooperación formal transfronteriza en Europa, con la puesta en marcha de la Iniciativa Comunitaria Interreg (1990), el presente análisis exploratorio plantea un modelo de análisis de la cooperación de "segunda generación" a través del estudio de la experiencia de la Red Ibérica de Entidades Transfronterizas (RIET), que desarrolla su actividad en el espacio de frontera de España y Portugal. El caso de la RIET nos permite constatar la construcción de un modelo de cooperación ampliado (formal-informal) y reforzado (isopoliteia y sympoliteia), denominado Europolis, basado en prácticas de gobernanza transfronteriza local, que contempla los procesos de cooperación transfronteriza desde ópticas de diversidad y flexibilidad. Para analizar el modelo se toma como ejemplo la construcción de la isopoliteia y la sympoliteia practicada por las ligas de ciudades en la Grecia clásica, y se aplica a la realidad de las redes de ciudades que ponen en marcha proyectos en las fronteras interiores de la Unión Europea, en nuestro caso, la RIET. Para ello, se estudia su path dependence desde el neoinstitucionalismo histórico, además de su influencia en la definición de problemas públicos transfonterizos y en la posterior conformación de las agendas públicas de las Cumbres Ibéricas, que España y Portugal celebraron los años 2013 y 2014.
\end{abstract}

Palabras clave: Europolis; isopoliteia; sympoliteia; cooperación informal; redes locales; RIET; gobernanza multinivel y transfronteriza.

\footnotetext{
* Parte de los resultados expuestos en este trabajo son resultado de la participación del autor Enrique José Varela Álvarez en el proyecto de investigación "Cooperación transfronteriza y [des]fronterización: actores y discursos geopolíticos transnacionales en la frontera hispano-portuguesa" [CSO2012-34677 I+D], financiado por el Ministerio de Economía y Competitividad (2012-2016). Deseamos agradecer a los evaluadores de la revista sus aportaciones a lo largo del proceso de revisión, que, sin duda, han redundado en una mayor calidad del trabajo y de sus conclusiones.
} 


\title{
Building Europolis on the Basis of Local "Informal” Cooperation in European Cross-Border Spaces: The Case of RIET
}

\begin{abstract}
After twenty five years of cross-border formal cooperation in Europe with origins in the landmark launch of the Communitarian Initiative Interreg (1990), this exploratory analysis offers a model for the study of "second generation" cooperation based on the experience of the Red Ibérica de Entidades Transfronterizas (RIET) that operates in the border space between Spain and Portugal. The case of RIET enables the observation of a model of widened (formal-informal) and reinforced (isopoliteia and sympoliteia) cooperation. Under the name of Europolis, it is based on practices of cross-border local governance that address the processes of cross-border cooperation from diversity and flexibility standpoints. For the examination of the model, the construction of the isopoliteia and sympoliteia put in practice by the leagues of classical Greek cities is taken as an example; and, in turn, applied to the realities of the cities' networks that boost the development of projects across the European Union's inner borders - in our case, the RIET. To this end, their path-dependence is explored from a historical neo-institutionalist perspective, as well as their influence in the definition of cross-border public issues and the political agendas of the Iberian Summits, which brought Spain and Portugal together in the years 2013 and 2014.
\end{abstract}

Key words: Europolis; isopoliteia; sympoliteia; informal cooperation; local networks; RIET; multi-level and cross-border governance.

\section{Construindo Europolis a partir da cooperação local “informal” nos espaços de fronteira da Europa: o caso da RIET}

\begin{abstract}
RESUMO
Depois de vinte cinco anos do início da cooperação transfronteiriça formal na Europa, com o lançamento da Iniciativa Comunitária Interreg (1990), esta análise exploratória apresenta um modelo de análise da cooperação de "segunda geração", através do estudo da experiência da Rede Ibérica Entidades Transfronteiriças (RIET), que opera na zona fronteiriça entre Espanha e Portugal. O caso da RIET nos permite observar a construção de um modelo de cooperação ampliado (formal-informal) e reforçado (isopoliteia e sympoliteia), chamado Europolis, com base em práticas de governança local transfronteiriça, que inclui os processos de cooperação transfronteiriça na perspectiva de diversidade e flexibilidade. Para analisar o modelo é tomado como um exemplo a construção da isopoliteia e da sympoliteia praticada pelas ligas de cidades na Grécia clássica, e se aplica à realidade das redes de cidades que implementam projetos nas fronteiras internas da União Europeia, no nosso caso, a RIET. Para esse fim, estuda-se seu path dependence na ótica do institucionalismo histórico, além de sua influência sobre a definição de problemas públicos transfronteiriços e a subsequente formação de agendas públicas das Cúpulas Ibéricas, que Espanha e Portugal celebraram nos anos de 2013 e 2014.
\end{abstract}

Palavras-chave: Europolis; isopoliteia; sympoliteia; cooperação informal; redes locais; RIET; governança multinível e transfronteiriça.

\section{REFERENCIA NORMALIZADA}

Domínguez Castro, Luis, y Varela Álvarez, Enrique José (2015) “Construyendo Europolis a partir de la cooperación local «informal» en los espacios transfronterizos de Europa: el caso de la RIET". Geopolítica(s). Revista de estudios sobre espacio y poder, vol. 6, núm. 2, 225-246. 
SUMARIO: 1. Presentación: la Grecia clásica y el estudio de la cooperación transfronteriza. 2. La RIET, entre la "cooperación formal" y la "cooperación informal". 2.1. La path dependence de la RIET. 2.2. La RIET en las Cumbres Hispano-Lusas del 2013 y 2014. 3. Conclusiones: Hacia una nueva Europolis a partir de la cooperación transfronteriza local e informal. Bibliografía.

\section{Presentación: la Grecia clásica y el estudio de la cooperación transfronteriza}

Los expertos reunidos por la Comisión Europea para reflexionar sobre las ciudades del mañana, concluyeron, en 2011, que una de la líneas fuerza del futuro urbano pasa por encontrar nuevas fórmulas de gobernanza que permitan acompasar los rígidos límites administrativos de los municipios de hoy, con alternativas que no supongan, necesariamente, la creación de nuevas estructuras institucionales formales (Unión Europea, 2011: 93).

Es por ello, por lo que, en los últimos tiempos, diferentes instituciones y organismos comunitarios han aprobado importantes documentos que tienen como objetivo la necesidad de contar con agendas urbanas, tanto en el ámbito de la UE, como en los ámbitos nacionales, regionales y locales. Así, el Parlamento Europeo (2011) aprobó una resolución en la que se abogaba por reforzar la dimensión urbana de las políticas de la UE en la programación 2014-2020, solicitando expresamente la elaboración de una agenda urbana, e instando a una mayor cooperación intergubernamental a través de reuniones informales de Ministros responsables de las políticas urbanas a nivel nacional. El Comité de las Regiones (2014), dio aprobación a un dictamen en el que, entre otras cosas, se insta a la Comisión Europea para que elabore un Libro Branco sobre el tema en el que se incluya una definición clara del concepto de desarrollo urbano sostenible e integrado, y que establezca de manera inequívoca los objetivos que la UE desea alcanzar en este campo y en los diferentes ámbitos políticos. La Comisión Europea (2014), como primer paso en esa dirección, presentó en 2014 una Comunicación, seguida de un período de consulta pública entre todos los agentes y ciudadanos interesados, proceso que concluyó el 26 de septiembre de 2014. Por su parte, el Consejo de la Unión Europea ha creado un grupo de trabajo encargado de diseñar una visión común de las ciudades europeas en la que se preste especial atención a la posición de las ciudades en la Estrategia Europa 2020, este grupo debe presentar sus conclusiones a lo largo del año 2016.

Como podemos observar, en estos momentos, diversos actores implicados, incluida la propia Comisión Europea, parecen aceptar la evidencia de que el espacio natural de la cooperación es el local, urbano y/o rural, y/o de redes de ciudades, y que la metodología de acción es la gobernanza multinivel y transfronteriza (Comisión Europea, 2014; Morata, 2004: 19-50; Varela, 2013 y 2015), sobre todo en los espacios eurorregionales (Rojo y Varela, 2011: 1-17).

Al hilo de esta idea, el presente trabajo pretende ahondar en esas posibles alternativas locales no formales (Medina, 2006a: 18), o "cooperación informal" (Varela, 2015), de estructuras que permitan mejorar la cooperación transfronteriza a partir de 
nuevos sistemas de gobernanza local y transfronteriza que puedan articular mejor el territorio y hacer posible así un incremento real de la calidad de vida de los ciudadanos ${ }^{1}$. En este sentido, definiremos "cooperación informal" como las "prácticas de interdependencia" (Woodward y Jones, 2005: 236, citado por Lois y Cairo, 2011: 13), desarrolladas de forma cotidiana por la sociedad civil, organizada o de forma individual (ciudadanos-vecinos) de las ciudades grandes, de las villas medias o de las localidades más rurales, que se sitúan a uno y otro lado de un espacio transfronterizo $^{2}$. También a las prácticas de cooperación transfronteriza puestas en marcha por instituciones de carácter asociativo, empresarial y/o educativo, que son fruto de la tradición y de la cultura transfronteriza, y que no disponen, necesariamente, de un soporte jurídico y/o institucional para su funcionamiento. Este tipo de "cooperación informal" tiene lugar en espacios locales y se contrapone (tan sólo a veces se complementa) con las prácticas formales y materiales de la cooperación institucionalizada, producida en espacios territoriales y político-administrativos regionales y/o estatales (Cancela, Cordal, Domínguez y Varela, 2012: 61-73; Varela, Cancela y Cordal, 2015: 77-95).

El planteamiento sobre "cooperación informal", parte de la teorización expuesta por el politólogo belga Paul Magnette (1999) en torno a los conceptos de gobernanza (Magnette, 2003) y ciudadanía (Magnette, 2005) en la Unión Europea; desarrolladas históricamente en las prácticas constitucionales de las polis de la Grecia clásica y helenística, y visible en los procesos de isopoliteia y sympoliteia que tuvieron lugar entre sus instituciones de gobierno (Pascual, 2007) ${ }^{3}$. El empleo de estos conceptos clásicos y más contemporáneos, pretenden ayudar a definir nuestro objeto de estudio, Europolis, en el contexto de la "cooperación informal" de base local en la Unión Europea.

En el caso de la isopoliteia y la sympoliteia, nos situamos ante dos prácticas constitucionales clásicas que tuvieron su origen, en la mayor parte de los casos, en enfrentamientos bélicos, con la finalidad principal de creación de una alianza militar. No obstante, siempre han tenido un fondo religioso y cultural común. También es cierto que muchas de ellas acabaron siendo instrumento hegemónico de la polis más fuerte, salvo en el período helenístico en el que se crearon diversas alianzas para enfrentarse, precisamente, a la hegemonía macedonia. A pesar de todo, cree-

\footnotetext{
${ }^{1}$ El momento actual es el adecuado, ya que podemos echar la vista atrás y establecer algunas conclusiones en torno a la cooperación territorial europea, especialmente a la transfronteriza, que cumple de la mano de Interreg 25 años (1990-2015), y que representa todo un modelo global de "cooperación formal".

2 En adelante denominaremos como "ciudadanos" a cualquier vecino/a de un territorio local, sea este urbano o rural, que es, en todo caso, sujeto de derechos y obligaciones en un espacio democrático.

${ }^{3}$ La isopoliteia consistía en un vínculo horizontal entre las ciudades-estado por el que los ciudadanos de una polis podían disfrutar de los derechos cívicos de otra mediante un pacto de reciprocidad. Por su parte, la sympoliteia nacía del pacto creador de estructuras e instituciones compartidas y democráticas. En realidad la segunda no implica la existencia de la primera, sino que tenían vías diferentes (Pascual, 2007: 177).
} 
mos que ambos conceptos pueden ser un buen punto de partida para el análisis de la configuración de nuevas institucionalidades de la "cooperación informal" o de cooperación colaborativa, que se crean a partir de estructuras informales de cooperación entre localidades fronterizas de dos o más estados de la UE. En el contexto de la Europa unida, el concepto de localidad fronteriza debe alejarse del hecho físico de la separación territorial. Por lo tanto, la proximidad a la linde de demarcación ya no debe ser el elemento discriminador esencial en el marco de configuración de un mercado único superador de muchas de las barreras tradicionales que estaban asociadas a la frontera. En consecuencia, las Europolis comunitarias van a tener como espacio geográfico las NUTS III fronterizas y, también en muchos casos, las NUTS III adyacentes siguiendo la nomenclatura de los propios programas de cooperación territorial. Evidentemente, el papel de las ciudades comprendidas en este espacio va a ser diferente. La práctica de la isopoliteia será una aspiración mucho más sentida cuanta mayor proximidad exista y menguará a medida que la distancia y la falta de contactos regulares se incremente. No obstante, no será infrecuente el interés de ciudades alejadas, por participar en estructuras de sympoliteia, asumiendo incluso su liderazgo, con el fin de incrementar su visibilidad y centralidad políticas ${ }^{4}$.

En este sentido, la experiencia más reciente de décadas de cooperación transfronteriza en Europa (Domínguez y Pires, 2014) nos muestra que son muchas las lecciones que tenemos que aprender de las ciudades, grandes, medianas y pequeñas, de sus redes y asociaciones. Porque la Unión Europea es también una unión de territorios y comunidades sociopolíticas locales que definen, a su vez, una "Europa de las Ciudades" (Goldsmith, 1994), y que son, en todo caso, actores plenos de la cooperación transfronteriza (Hocking, 2004).

En efecto, la UE ha consagrado, desde el Tratado de Roma, la isopoliteia en tanto en cuanto establece el principio de la no discriminación de los ciudadanos comunitarios en relación con los nacionales - cierto que este principio sólo rige para los inquietos que ejercen la movilidad entre las diferentes fronteras nacionales (Middelaar, 2013: 406)—; por su parte, cuando elegimos a los miembros del Parlamento Europeo estamos practicando la sympoliteia.

Afortunadamente las urgencias militares no están presentes ya en la actualidad en las agendas públicas de los Estados que conforman la Unión Europea, por lo que en las agendas públicas de los Estados miembros sólo se contemplan, de forma cíclica, las cuestiones de seguridad transfronteriza cuando se producen crisis

\footnotetext{
${ }^{4}$ Para la mejor comprensión de este fenómeno, puede resultar ilustrativo el estudio de los casos de las Eurociudades de la frontera ("raia") de España con Portugal (Lois y Cairo, 2015), tanto en la parte dividida por el río Miño/Minho ("raia húmeda"; Lois, Limón y Díaz, 2015), como en el resto de la frontera hispanolusa ("raia seca"; De la Macorra y Cano, 2011; Lois, 2013); así como el caso singular de la ciudad de Porto en el contexto de la trama urbana de la Eurorregión Galicia-Norte de Portugal (Domínguez, 2004).
} 
migratorias como la que está viviendo Europa en el año $2015^{5}$. Así, la sympoliteia de las ciudades resultaría ahora de la necesidad de ejercer un lobbying eficaz, tanto ante las autoridades nacionales como ante las comunitarias, con el objetivo de mejorar la calidad de vida de la ciudadanía e incrementar la visibilidad y participación política de las instituciones locales, en un contexto global más favorable para el conjunto de sus actores. En efecto, en la última década, el proceso de urbanización de Europa en particular y del mundo en general, ha experimentado un rápido crecimiento. Las ciudades, ahora más que nunca, se presentan como el epicentro del crecimiento y la creación de empleo; lugares de intercambio, cultura e innovación, en donde las oportunidades y los retos para el crecimiento sostenible conviven, no siempre en equilibrio.

No obstante, cualquier intento de adaptar experiencias de isopoliteia o de sympoliteia a la realidad de las ciudades de frontera en la UE debe partir de la asimetría existente entre ellas. En efecto, los ordenamientos jurídicos de cada país confieren diferentes marcos de competencias a sus ciudades y distintos órganos de gobierno que influyen en su capacidad para gestionar las políticas públicas y en su autonomía financiera. Además, el tamaño de las mismas es importante a la hora de determinar la amplitud de su margen de maniobra como actor eficiente en la cooperación transfronteriza. El concepto de Europolis que planteamos, intenta acomodar la asimetría territorial, política, administrativa, financiera y sociodemográfica de las ciudades europeas, a la oportunidad que la cooperación ofrece para mejorar la calidad de vida de sus ciudadanos y los sistemas de gobernanza. Esto es así porque, siendo asimétricos los poderes locales son mucho más homogéneos que los poderes regionales y, por supuesto, tienen una libertad de acción exterior (paradiplomacia) impensable en los poderes nacionales. Cualquier manifestación o declaración de un miembro del gobierno nacional compromete a su país, mientras que un cargo electo local puede emitir opinión sobre aspectos relacionados con la acción exterior de su país sin comprometerlo de forma grave. El nivel local resulta pues idóneo para establecer una nueva sympoliteia europea de carácter informal y, sin duda, es quien mejor conoce las necesidades y oportunidades de isopoliteia, aunque sus competencias no les permitan, en muchas de las ocasiones, hacerlas operativas.

Nuestra propuesta de Europolis se concreta en el análisis de las redes de ligas de ciudades unidas por un interés común y dotadas de una enorme flexibilidad, que implica que su carácter pueda ser permanente o temporal, tener personalidad jurídica propia o emplear subsidiariamente la que tienen sus partes integrantes, tener

\footnotetext{
${ }^{5}$ El fenómeno de la presión migratoria en las fronteras exteriores de la Unión Europea, lejos de tener un carácter coyuntural, se manifiesta como un fenómeno estructural sobre todo a partir de la última década del pasado siglo. Este proceso tiene su reflejo en la evolución de los instrumentos normativos comunitarios (convenciones o regulaciones) sobre movilidad y control fronterizo, que van de Schengen a Dublín (1985 y 2013).
} 
predominio de ciudades medianas o pequeñas en su seno, englobar amplios espacios rurales o carecer de ellos, configurarse a través de ligas parcas en su número de ciudades miembro o ser generosas en el mismo. Europolis no se entiende como una única organización de ciudades, sino como la sympoliteia de varias de ellas. La diversidad es una de sus señas de identidad, junto con la flexibilidad señalada. Flexibilidad que va más allá de sus características constitutivas u organizativas y que también abarca su campo de actuación. Europolis aplica el método de cooperaciones reforzadas que no obligan a todos sus miembros a implementar las mismas políticas y poder entrar y salir de ellas en función de sus intereses, pero con la isopoliteia como horizonte y meta compartida. La flexibilidad organizativa de Europolis permite abrir la red a otros agentes económicos y sociales de los espacios de frontera, tales como las organizaciones empresariales y las instituciones del conocimiento, representadas por los centros de educación superior; incluso, por extensión, a la ciudadanía en su sentido más amplio. Evidentemente, el espacio fronterizo es un lugar privilegiado para la puesta en marcha de Europolis, como una nueva institucionalidad transfronteriza de carácter local, que puede hacer evolucionar la cooperación hacia otro modelo, que algunos autores denominan de "segunda generación" (Medina, 2012: 9-18).

El caso de la Red Ibérica de Entidades Transfronterizas (RIET), entidad conformadora de una suerte de nueva institucionalidad de cooperación informal e inmaterial, puede asimilarse a este modelo de Europolis, anteriormente expuesto. Por ello, el objetivo del presente trabajo consiste en constatar si las aportaciones de la RIET a la cooperación formal e informal, su construcción institucional, su influencia en el entorno transfronterizo hispano-luso, permitiría avanzar soluciones a los problemas y agendas actuales de los espacios de frontera, a partir del modelo de Europolis que hemos presentado; cuestiones que desarrollamos en los apartado siguientes.

\section{La RIET, entre la “cooperación formal” y la “cooperación informal”}

Las ciudades de frontera se han dado cuenta de su importante capital simbólico y unidas por el cemento de sus afinidades culturales, económicas y sociales han puesto en marcha una fórmula de influencia en sus respectivos gobiernos regionales (como en el caso español), nacionales e instituciones comunitarias, capaz de conferirles una centralidad, que hace mucho tiempo habían perdido.

En este nuevo marco informal, la finalidad de la sympoliteia de los poderes locales fronterizos es explícita y tiene un objetivo principal ${ }^{6}$ : hacer lobby, influir en la

\footnotetext{
${ }^{6}$ Existen otro tipo de objetivos, más concretos y tangibles, que pasan por el interés de los actores locales por conseguir formar parte candidaturas de proyectos comunitarios y alcanzar fondos destinados a la coopera-
} 
confección de las agendas públicas de sus respectivos gobiernos nacionales, más concretamente, y en el caso que nos ocupa de la Red Ibérica de Entidades Transfronterizas (RIET), en la agenda de las Cumbres Ibéricas entre España y Portugal de los años 2013 y 2014.

El acquis de gestión de políticas públicas que ha ido generando el proceso de integración europea, permite extraer algunas reflexiones sobre el funcionamiento de estas estructuras informales de cooperación. En efecto, puede abordarse ese funcionamiento y sus objetivos desde la perspectiva neorrealista de las ganancias relativas o desde la institucionalista de las ganancias absolutas. En el primer caso, lo importante es mantener los equilibrios y ello obligaría a reducir el campo de actuación del lobby para no ponerlos en peligro, dada la heterogeneidad de los miembros de la entidad de cooperación; lo simbólico tendería a predominar sobre lo operativo. En el segundo caso, lo importante es que todos los miembros ganen, aunque unos, los más dinámicos y capaces, ganen más. Los actores más pequeños (territorial y poblacionalmente hablando) pueden lograr grandes avances en este escenario, que por su escaso poder de influencia subnacional tendrían dificultades para alcanzar por separado. Los grandes (generalmente ciudades), amén del capital simbólico conseguido, pueden ser considerados portavoces de colectivos más amplios, lo que siempre reforzará su posición negociadora ante los posibles interlocutores regionales, nacionales y comunitarios.

Partiendo del objetivo enunciado en el apartado anterior, a continuación vamos a aplicar la propuesta de análisis de Europolis a una realidad institucional concreta, como es el caso de la Red Ibérica de Entidades Transfronterizas (RIET) en el espacio de la frontera entre España y Portugal, a partir de las siguientes preguntas: ¿es la RIET una nueva institución de "cooperación informal"?, ¿se ajusta al perfil de Europolis que proponemos?; ¿qué ha aportado desde su constitución a la cooperación transfronteriza hispano-lusa?

A tal fin, se van a analizar los procesos de cooperación formal e informal de la RIET, a partir del estudio de su path dependence y de su papel e influencia en la conformación de las agendas públicas que se desarrollaron en las Cumbres Ibéricas celebradas en los años 2013 y 2014. Para lograr dichos objetivos se realizará un análisis exploratorio de las fuentes documentales primarias (Selltiz, Wrightsman y Cook, 1980: 134-148; Manheinn y Rich, 1988: 51-65) que tienen relación con la RIET, especialmente sus declaraciones fundacionales y los dosieres de prensa emitidos por su equipo de gestión, en relación con los diferentes temas que la RIET considera estratégicos para su organización. Este método cualitativo se combina con la técnica del estudio de casos (Eckstein, 1975: 79-138), apropiado para entender la

ción, en cualquiera de las versiones que marca la Cooperación Territorial Europea. Este objetivo no forma parte del proceso de análisis de la investigación. 
realidad de una entidad de cooperación novedosa en el panorama de las instituciones transfronterizas en el contexto dado de la frontera hispano-lusa. El enfoque teórico que se va a aplicar al estudio de caso de la RIET, se corresponde con la perspectiva institucionalista, adaptada a partir de las propuestas del neoinstitucionalismo histórico (Steinmo, 2013) al espacio transfronterizo (Peña, 2007; Varela, 2010), lo que nos puede ser utilidad para explorar nuevos modelos de cooperación territorial desde el análisis de un mix "líquido" (Bauman, 2009) que surge tras la crisis de los modelos formales institucionales, y que obliga a la (re)creación de los modelos informales, hegemónicos en los años previos a la entrada de España y Portugal en la Comunidad Económica Europea de los años 80 (Domínguez y Pires, 2014; Varela, Cancela y Cordal, 2015).

\subsection{La path dependence de la RIET}

Para entender esta Europolis del siglo XXI, y los conceptos de sympoliteia e isopoliteia adaptados a las propuestas de la gobernanza multinivel y transfronteriza, tomamos como referencia la Red Ibérica de Entidades Transfronterizas (RIET), estructura informal novedosa que cuenta con un cierto grado de institucionalización en los procesos de cooperación territorial entre España y Portugal.

Porque las preguntas claves sobre la RIET, su papel en esta nueva institucionalidad de la cooperación territorial formal e informal, tienen que ver con el por qué y cómo se gestó la creación de esta estructura de cooperación territorial; cuál fue su path dependence en el contexto de la cooperación multinivel en la Unión Europea de los inicios del siglo XXI.

Sin duda, el hito prefundacional de la RIET tuvo lugar durante la celebración del Seminario Cooperação Transfronteiriça de Segunda Geração los días 4 y 5 de febrero de 2008 en Guimarães (Portugal); en un foro organizado conjuntamente por el Instituto Financeiro para o Desenvolvimento Regional de Portugal ${ }^{7}$ y por el Eixo Atlântico do Noroeste Peninsular ${ }^{8}$. El Instituto portugués quería publicitar las buenas prácticas y logros de la etapa cubierta por el INTERREG IIIA, mientras que

\footnotetext{
${ }^{7}$ El IFDR era, en ese momento, el organismo público portugués que ejercía como autoridad de certificación de los fondos FEDER, Fondo de Cohesión y programas de cooperación territorial europea. En la actualidad, y desde 2013, se ha fusionado con el Instituto de Gestão do Fundo Social Europeu y con la Estrutura de Missão Observatório do QREN, dando lugar a la Agência para o Desenvolvimento e a Coesão, responsable de la coordinación de las políticas de desarrollo regional y de la coordinación general de los fondos europeos estructurales y de inversión.

${ }^{8}$ El Eixo Atlântico do Noroeste Peninsular nace en 1992, con la personalidad jurídica de una asociación privada portuguesa, como estructura de cooperación entre los principales municipios urbanos de Galicia y del Norte de Portugal. En 2009 contaba con 34 socios, 17 de cada una de las dos regiones; en el año 2015 son 38, incluidas las Diputaciones Provinciales de Coruña y Lugo.
} 
la asociación de municipios transfronterizos pretendía usar el encuentro como plataforma para tejer su sympoliteia local, a partir de la cual tener mayor protagonismo en la arena comunitaria de los poderes locales y sus lobbies.

Así es como nace la RIET, creada un año después, el 23 de junio de 2009, durante el transcurso de otro seminario sobre la cooperación transfronteriza en la frontera de España y Portugal (Espacio Ibérico, las ciudades en el espacio transfronterizo del oeste peninsular), celebrado esta vez en Cáceres y que da lugar a la Declaración de Cáceres, protocolo fundacional de la RIET. La RIET surge pues con la participación de doce entidades transfronterizas, todas ellas dotadas de personalidad jurídica (bien por sí mismas, bien a través de sus integrantes). Con posterioridad se han ido incorporando otras entidades hasta completar el mapa territorial de la frontera, de manera que desde el 27 de noviembre de 2012 pasan a formar parte de la RIET la mayoría de las asociaciones empresariales presentes en la frontera hispano-lusa, lo que la confiere singularidad dentro del contexto europeo. Finalmente, desde el 24 de noviembre de 2014, se han incorporado seis universidades ${ }^{9}$ cerrando, así, la "triple hélice" de empresarios, centros superiores de investigación y administraciones públicas (Mapa 1).

Tras la incorporación de las organizaciones empresariales, la RIET se autodefine, un tanto pomposamente, como "el mayor lobby de frontera, público y privado, de Europa" (RIET, 2012). En la Asamblea General, celebrada el 15 de octubre de 2013 en Ourense, la RIET señala su pretensión de "contar con voz única ante los gobiernos de España, Portugal y de la Unión Europea en el momento en que se negocian en Bruselas los fondos para el periodo de programación 2014-2020" (Eixo Atlântico, 2013). De hecho, la Comisión Luso-Española, órgano preparatorio de dichas cumbres, admitió, en su reunión del 4 de abril de 2013 en Vigo, el establecimiento de un canal permanente de comunicación entre ella y la RIET (Eixo Atlântico, 2013). Este canal existe desde hace mucho tiempo con los gobiernos de las Comunidades Autónomas, pero es la primera vez que se aplicaba a un foro de autoridades locales.

En origen, pues, se trataría de una liga de ciudades, o mejor de una liga de ligas de ciudades previamente constituidas, a la que se incorporan agentes sociales y otras organizaciones de la sociedad civil. De esta forma, se podría decir que la RIET es un modelo de Europolis que practica una sympoliteia adaptada a los nuevos tiempos de gobernanza multinivel de carácter horizontal, esto es con la participación de actores públicos y actores privados, en ambos casos no estatales (KohlerKoch y Rittberger, 2006), a los que todavía sigue siendo ajena la participación de los ciudadanos (Magnette, 2003).

\footnotetext{
${ }^{9}$ Vid. Europa Press (2014): "Seis universidades rayanas se unen a la Red Ibérica de Entidades Transfronterizas para fomentar la cooperación". Disponible en URL: <DOSSIER_RIET_17_19_NOV_14_OP_2.pdf $>$. Consultado el 7 de abril de 2015.
} 
Mapa 1. El espacio transfronterizo de la RIET (2014)

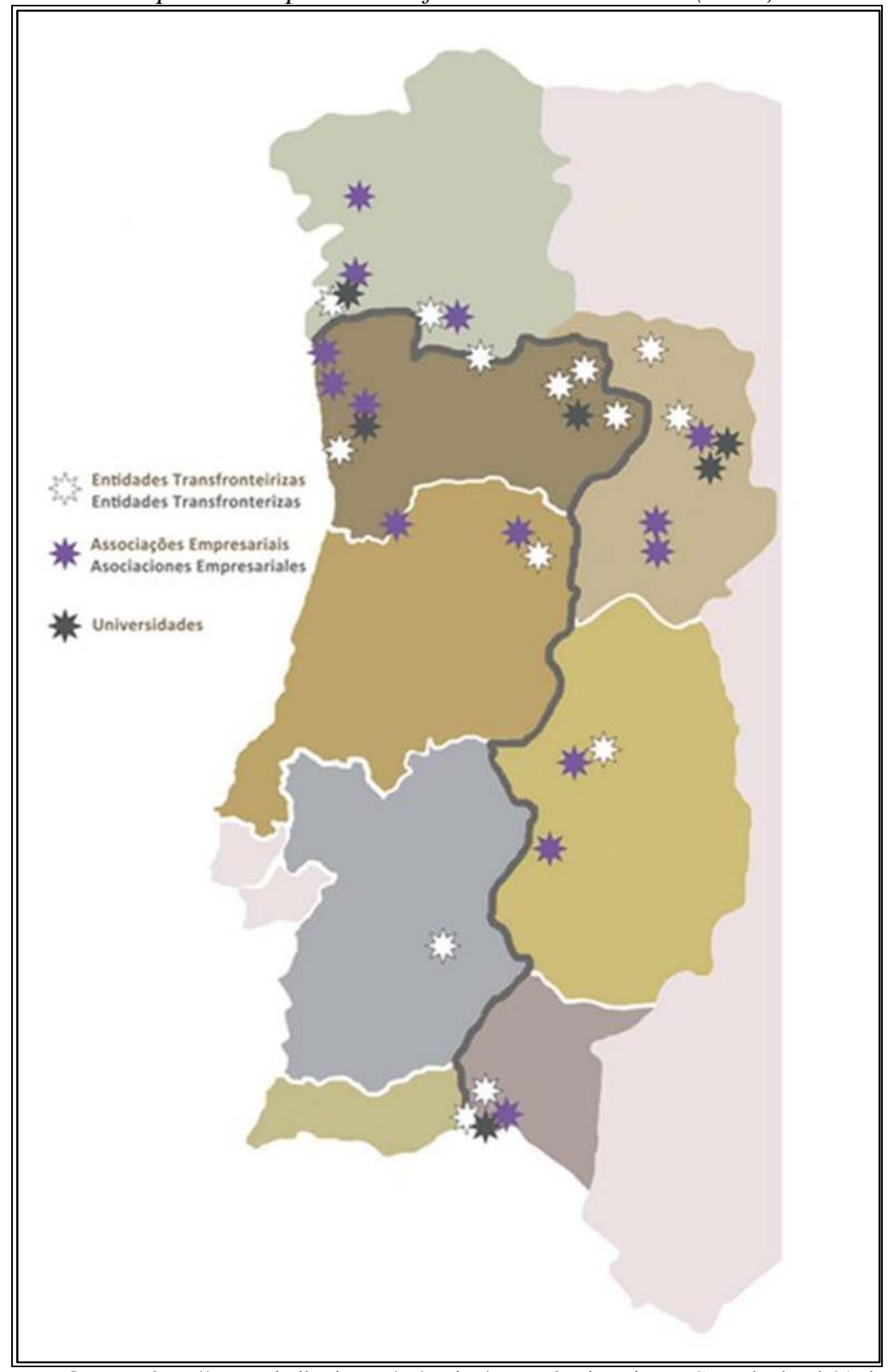

Fuente: RIET [URL: <http://www.rietiberica.eu/es/socios/mapa-de-situacion>. Consultado el 20 de diciembre de 2014]. 
En realidad, la RIET se centra en las acciones de sympoliteia, en cuanto que liga de ciudades, dejando las labores de isopoliteia a la actuación de algunos de sus miembros, muy especialmente las Eurociudades de Chaves-Verín y de BadajozElvas (Lois, Limón y Díaz, 2015).

En Europa, el caso de las ligas de ciudades, o redes de ciudades, no suponía ninguna novedad, ya que se contaba con estructuras similares, que agrupaban entidades urbanas y rurales de cooperación, como la MOT francesa o las City Twins bálticas. Sin embargo, en la para algunos autores considerada la frontera más antigua de Europa $^{10}$ (Medina, 2006b; López, 1998), la iniciativa de la RIET, como nueva entidad de cooperación de "segunda generación" (Medina, 2012), va a ser impulsada por el Eixo Atlântico do Noroeste Peninsular, actor local transfronterizo hispanoluso que lleva funcionando en el ámbito de la cooperación transfronteriza local, de forma ininterrumpida, desde el año 1992.

En efecto, el Eixo había alcanzado el suficiente grado de madurez organizativa como para entender que las ciudades que no eran capitales de sus respectivos estados, o no eran centrales por su peso económico o geoestratégico, tenían escasa capacidad de lobby en una Bruselas que cada vez condicionaba más las políticas públicas a todos los niveles. Al mismo tiempo, los franceses observaban con preocupación su creciente pérdida de influencia en el Este europeo y las dificultades para liderar lobbies territoriales, al no lograr la Conferencia de Regiones Periféricas y Marítimas (CRPM) sobreponerse a la Asociación de Regiones Fronterizas de Europa (ARFE), dominada por los germanos, y con una Asociación de Regiones de Europa desactivada. A principios de la década pasada, la Mission Opérationnelle Transfrontalière (MOT) ${ }^{11}$ estaba buscando socios para conformar un nuevo lobby transfronterizo, con fuerte componente urbano, que pudiera singularizarse en la arena comunitaria como cabeza de un nuevo grupo de presión. En este contexto, se crea EUROMOT, como una red europea de autoridades locales transfronterizas que

\footnotetext{
${ }^{10}$ En torno a esta afirmación no existe unanimidad académica en los estudios sobre fronteras en Europa, pudiendo encontrar autores que defienden argumentos a favor y en contra (Trillo-Santamaría y Valerià, 2014); por ello, es una cuestión en la que no se va a profundizar en el presente trabajo, al suponer una dimensión de análisis colateral al problema investigado.

${ }^{11}$ La MOT fue creada por el gobierno francés, en 1997, como entidad coordinadora de los distintos actores que intervienen en la cooperación territorial a lo largo de las nueve fronteras nacionales que tiene el país, al tiempo que también ejercía como gabinete técnico de apoyo para la generación de estrategias y presentación de proyectos de cooperación territorial, así como para la formación de agentes transfronterizos. Esta última actividad ya tenía, en los años previos al seminario de Guimarães, su propia red a través de Interform, una red europea de formación y de investigación de prácticas transfronterizas, financiada por el programa INTERACT 2000-2006. Interform, liderada por la MOT englobaba a universidades y centros superiores de Francia, Italia (Génova), Suiza (Ginebra) y España (Deusto). En la actualidad, la MOT, en asociación con ARFE, ha impulsado The Transfrotier Euro-Institut Network (TEIN) con la presencia de doce miembros, pertenecientes a fronteras de catorce países europeos.
} 
acogía a la propia MOT, al Eixo Atlântico y a la City Twins ${ }^{12}$, en el marco de los Rencontres Européennes organizados por la MOT, en Lille, los días 8 y 9 de noviembre de 2007.

El problema es que los objetivos de los tres socios fundadores de esta red no eran los mismos. City Twins aspiraba a tener continuidad como tal estructura gracias a la presentación de una candidatura de la red al programa INTERREG IVC. La MOT coincidía también en el interés por obtener financiación comunitaria pero quería además, como el propio nombre de la red indicaba, ejercer el liderazgo de las autoridades locales transfronterizas a lo largo de la Unión Europea ${ }^{13}$. Por su parte, el Eixo encontraba en esta iniciativa su primera experiencia transnacional comunitaria y comprendió que para tener peso en cualquier red lobbista comunitaria y poder llegar a ser un interlocutor de la Comisión y demás instituciones europeas, era necesario contar con una plataforma de apoyo más fuerte, algo así como una MOT ibérica a lo largo de la frontera hispano-portuguesa. En realidad, el seminario de Guimarães de $2008^{14}$ fue el equivalente al celebrado en Lille, que había servido como lanzamiento de EUROMOT; estructura que para entonces ya estaba olvidada.

Como se ha podido constatar, el surgimiento y evolución institucional de la RIET se produce en un contexto de competencia por los fondos comunitarios, con el objetivo de ampliar la posición de lobby de las entidades originales, especialmente del Eixo Atlântico, en Europa. De hecho, la RIET fue miembro fundador de la Conference of European Cross-border and Interregional City Networks (CECICN), en abril de 2010, que en la actualidad agrupa a 9 grandes ligas de ciudades que cubren la práctica totalidad del espacio geográfico de la UE. Esta perspectiva ha ido completándose con la construcción de nuevas "líneas de negocio", como es la creación de la agencia de desarrollo transfronterizo o el "Observatorio de Frontera" - incluye el Centro de Estudios y el Centro de Documentación-, que acaban por consolidar al Eixo y a la RIET en 2013 y 2014, como los actores de la cooperación transfronteriza más activos de la "raia" hispano-lusa. Todo ello se expone a

\footnotetext{
${ }^{12}$ Esta estructura de cooperación nace en 2006, al calor del programa INTERREG IIIC, con cinco socios bálticos que representan a ciudades hermanadas y vecinas entre sí: Imatra-Svetogorsk (Finlandia-Rusia); Narva-Ivangorod (Estonia-Rusia); Frankfurt Oder-Slubice (Alemania-Polonia); Görliz-Zgorzelec (Alemania-Polonia) y Tornio-Haparanda (Finlandia-Suecia). En la actualidad la asociación está conformada por los tres primeros socios y Valka-Valga (Letonia-Estonia).

${ }^{13}$ De hecho, una de las escasas concreciones de EUROMOT fue la publicación, en abril de 2008 del Manifiesto por la Cooperación Transfronteriza en Europa, que puede verse en URL: $<\mathrm{http} / / / \mathrm{www} . \mathrm{cvce} . \mathrm{eu} / \mathrm{en} / \mathrm{obj} / \mathrm{manifesto}$ for_cross_border_cooperation_in_europe_april_2008-en-92fb0898a949-411e-a919-577dea6717b9.html>. Consultado el 19 de enero de $\overline{2015}$.

${ }^{14}$ La puesta en escena tuvo su importancia. En efecto, el seminario fue inaugurado por sendos ministros de los gobiernos nacionales de Portugal y España y bendecido por el Director de Cooperación Territorial de la DG Regio. Asistieron representantes de las ocho regiones transfronterizas y de sus respectivas estructuras de cooperación, así como el Director General de la MOT.
} 
continuación a través del análisis de la construcción de la agenda de las dos últimas Cumbres Hispano-Lusas.

\subsection{La RIET en las Cumbres Hispano-Lusas del 2013 y 2014}

Llegados a este punto, parecen pertinentes las siguientes preguntas en relación con la RIET y su papel en la conformación de una nueva institucionalidad de la cooperación transfronteriza: ¿ha resultado útil la labor de lobby de la RIET frente a sus ejecutivos nacionales?; ¿se puede hablar de influencia de la RIET en la Cumbres Hispano-Lusas?

Aunque es pronto para obtener conclusiones, sí podemos plantear una línea de investigación a partir de la hipótesis de que la RIET pretende convertirse en un actor de la cooperación transfronteriza, a través de una estructura que combina lo formal e informal, con una trama organizativa que intenta recoger no sólo a entidades conformadas por poderes locales sino también a otras representativas del tejido empresarial y de las universidades; y siempre con un primer objetivo claro en el horizonte: ser un interlocutor con influencia en todo el territorio de frontera de España y Portugal. El segundo objetivo, de más largo recorrido, consistiría en alcanzar el mismo estatus en la arena comunitaria.

A la luz de los resultados de las Cumbres Hispano-Lusas de los años 2013 y 2014, es posible contrastar, por ejemplo, que tras la aprobación de una relación de peticiones a la Cumbre, por parte de la Asamblea General de la RIET en $2013^{15}$, una serie de temas estratégicos han aparecido en la agenda política de los Gobiernos de España y de Portugal. En este sentido, el 25 de marzo de 2014, la Comisión Hispano-Lusa se reunía con la RIET para conocer sus demandas. Las dos más importantes, sin duda, eran el plan de crecimiento de la frontera y el plan de movilidad; como veremos, ninguna de las dos se menciona en la Declaración Conjunta final de la Cumbre ${ }^{16}$.

\footnotetext{
${ }^{15}$ Peticiones a la Cumbre, aprobadas por la Asamblea General de la RIET en 2013: "Que los gobiernos español y portugués acuerden garantizar la navegabilidad del río Miño entre A Guarda y Caminha. La Elaboración de un Plan de Movilidad Transfronteriza. La Elaboración de un plan de crecimiento económico de la frontera que impulse, entre otros, una plataforma conjunta de los correos de ambos países e impulse la armonización fiscal. La Creación de un portal institucional conjunto que favorezca las tramitaciones administrativas entre ambos países. La Creación de una entidad conjunta coordinadora de las emergencias que actúe en casos de incendios, catástrofes, accidentes graves o cualquier otra emergencia en los territorios de frontera" (Eixo Atlântico, 2013).

${ }^{16}$ Vid. Declaración Conjunta de la XXVII Cumbre hispano-lusa. 4 de junio de 2014. Disponible en URL: $<$ http://www.cencyl.eu/_FILES/Declaracion_Conjunta_XXVII_Cumbre_luso_espanol.pdf $>$. Consultada el 23 de Septiembre de $20 \overline{14}$.
} 
En general, en materia de cooperación transfronteriza la Declaración tiene una dinámica propia y más centrada en la actividad de los gobiernos nacionales (grandes líneas europeas de transporte y energía, universidades y Laboratorio Internacional Ibérico de Nanotecnología) o regionales (sanidad). No obstante, la importancia de un lobby no sólo se debe medir por lo que consigue realmente, sino por lo que parece que ha conseguido. Desde esta perspectiva, la capacidad de visibilidad comunicativa que tenga resulta decisiva.

En efecto, los medios de comunicación pueden trasladar la imagen de una gestión eficaz más allá de los resultados reales. Así, la RIET consigue presentar como logros propios de su presión algunas medidas acordadas en la Cumbre de 2013. En concreto, la prensa ${ }^{17}$ se hace eco de que la interoperabilidad de los sistemas para el pago de los peajes va a acabar con el problema de accesibilidad a las autovías de Portugal. Se trata de la transcripción, más o menos literal, de la noticia enviada desde el gabinete de prensa de la RIET, que los medios reproducen. Otro tanto cabe decir sobre la modernización del tren Oporto-Vigo. Ambos temas tienen relación directa con la movilidad transfronteriza, lo que viene a demostrar la estrategia de largo recorrido que sigue la RIET, apostando por la elaboración de un plan de movilidad transfronterizo cuando ya ha conseguido colocar en las agendas públicas de los dos gobiernos ibéricos aspectos muy relevantes de ese "futuro" plan, que dice estar elaborando. El hecho de que en la realidad, y a fecha de cierre del presente trabajo, no se haya solucionado definitivamente el problema de los peajes en las viejas SCUT (Sem Custo para o Utilizador) portuguesas o que la modernización del tren se haya quedado, por ahora, en un mero cambio de máquinas y nombre ("Tren Celta"), no impide dar visibilidad a la RIET y a sus integrantes, convertidos en defensores de una causa popular cuya falta de resolución se achaca a los gobiernos alejados de la realidad fronteriza.

Como acabamos de ver, la capacidad de resonancia mediática de la RIET descansa en la existencia de un gabinete de prensa (Torres, 2010), radicado en la organización que ostenta su secretariado general (Eixo Atlântico), con habilidad para filtrar noticias a las dos principales agencias peninsulares, EFE y Lusa. Pero, además, esa visibilidad se busca, sobre todo en el caso español, en los medios locales de las áreas donde las autoridades políticas que forman parte de la organización y ejercen funciones directivas en la misma, tienen su clientela electoral ${ }^{18}$. Todo ello

\footnotetext{
${ }^{17}$ Vid. El Periódico de Salamanca (2014): "El embajador de España en Portugal aboga por que se concluyan las conexiones ferroviarias entre ambos países". Disponible en URL: $<$ DOSSIER_RIET_17_19_NOV_14_OP 2.pdf $>$. Consultado el 7 de abril de 2015.

${ }^{18}$ Así, los resultados de la reunión entre la RIET y los miembros de la Comisión hispano-lusa, celebrada el 25 de marzo de 2014, se publican (con información de agencia) en medios de esas características, pertenecientes a las regiones de la presidencia, vicepresidencia y secretaría de la RIET, como: Tribuna Salamanca [Tribuna Salamanca.com. Puesto en línea el 25 de marzo de 2014. Disponible en URL: $<$ http://www.tribunasalamanca.com>. Consultado el 26 de marzo de 2014]; Tribuna Ávila; Zamoranews;
} 
no obsta para que la noticia interese a la prensa de tirada nacional, claro está, sirviendo para ampliar el ámbito de influencia de la organización ${ }^{19}$.

Hemos señalado que la RIET se centra en las acciones de sympoliteia, dejando las relacionadas con la isopoliteia a sus miembros. Este hecho se puede percibir con nitidez en el tipo de demandas estratégicas que formula a las Cumbres. En efecto, las infraestructuras son su gran baza mediática, ya que, por ejemplo, la prensa local acostumbra a titular sus noticias sobre las Asambleas de la RIET con referencias a vías de comunicación que se demandan y están en sus respectivos ámbitos de influencia territorial. Es más, en la propia web de la organización, las demandas acordadas en la Asamblea General, celebrada el 24 de noviembre de 2014 en Cáceres, aparecen presentadas en cuatro grandes espacios transfronterizos reafirmando su carácter de liga de ciudades, de Europolis, en la que cada parte reclama lo suyo y apoya lo de los demás; al mismo tiempo, existen elementos vertebradores comunes como es el caso de los corredores de mercancías ferroviarios Sines-Badajoz, SinesFerrol y Aveiro-Salamanca, elemento prioritario de las demandas para la Cumbre de 2015 (RIET, 2014).

Otro claro ejemplo de la importancia otorgada a la sympoliteia, por parte de la RIET, podemos observarlo en la destacada presencia del embajador de España en Portugal en la Asamblea General de la organización en el año 2014; este responsable político estatal se manifestaba entonces de forma rotunda sobre la paradiplomacia de los poderes locales: "la RIET es una forma de hacer política exterior" (Europa Press, 2014) o que "complementa de una manera muy eficaz la acción del Estado" (El Periódico de Extremadura, 2014).

Finalmente, una nueva "línea de negocio" que procura consolidar la sympoliteia de la Europolis RIET, es el anuncio de trabajar en la constitución de una interparlamentaria de diputados y eurodiputados de los territorios de la frontera ibérica, anuncio lanzado en la asamblea de la organización en el año 2014 (RIET, 2014).

Todo este background podría parecer escaso, pero es necesario tener en cuenta que la importancia de poder acceder a influir en las agendas de las Cumbres Ibéricas, una vez conseguido el estatus de interlocutor, sólo puede medirse en relación con los resultados de las mismas, que en la mayor parte de las ocasiones son visibles a largo plazo. Un ejemplo claro lo encontramos en el desarrollo de la movilidad transfronteriza a través del tren, donde se constata que el problema de la modernización del trayecto ferroviaria Oporto-Vigo se encuentra en la agenda de los actores

Atlántico Diario; Diario de Bergantiños; Diario de Arousa; Diario de Ferrol; El Ideal Gallego; Porto Canal. Sin embargo, las frías o inexistentes relaciones del gabinete de prensa con otros medios explica que la noticia no haya aparecido en La Voz de Galicia, Faro de Vigo o El Norte de Castilla. En línea con el argumento expuesto, no hay repercusión en la prensa local extremeña o andaluza, por ejemplo.

${ }^{19}$ La misma noticia anterior se difunde (también a través de agencia) en: Público, Expresso, Expansión o la Vanguardia. Incluso llega a publicarse de igual manera en el Jornal da Madeira. 
regionales (Xunta-CCDR-N) desde el año $1999^{20}$, pero no entra en la agenda de los gobiernos de España y Portugal hasta bien entrada la segunda década del presente siglo (2014).

\section{Conclusiones: Hacia una nueva Europolis a partir de la cooperación trans- fronteriza local e informal}

Construir modelos explicativos sobre la realidad sociopolítica y territorial, requiere de gran dosis de imaginación y de aún mayores esfuerzos científicos en el ámbito de las Ciencias Sociales.

La propuesta que presentamos en este trabajo gira en torno al futuro de la cooperación transfronteriza, con base en un pasado clásico y contemporáneo, y en un presente posmoderno de crisis sistémica en Europa, que anima a redoblar los esfuerzos por imaginar nuevas institucionalidades, nuevos actores y nuevos espacios y procesos de cooperación transfronteriza, que desborden el marco del siglo XIX en el que fueron diseñadas y aprovechen el caudal de innovación que aporta la experiencia europea de las últimas décadas.

Europolis supone una aproximación exploratoria a las instituciones, actores y procesos de la cooperación transfronteriza en la Europa de los últimos veinticinco años. A través de una base territorial local ampliada, se amplía la propuesta de la cooperación a todo tipo de actores con intereses en la mejora de la cohesión y la calidad de vida en los espacios de frontera; por supuesto los públicos, desde luego los privados, e incluso, como no, aquellos, de carácter individual o agrupado, que defienden los bienes comunes. Así, el espacio local articulado en torno a sus gobiernos, cede protagonismo a otros actores, creando redes heterárquicas que desbordan la clásica colaboración intergubernamental y multinivel.

De esta manera comienza a vislumbrarse una innovadora modalidad de gobernanza, la transfronteriza, de base local, más próxima a los problemas y soluciones de los ciudadanos - ya no sólo de sus instituciones-, que articula su acción a partir de procesos de tipo bottom-up y backward mapping, y que recuperan el espíritu tanto de la isopoliteia helénica como de aquella otra que tenía lugar en todas las fronteras de la Europa de posguerra y que pervivió hasta la entrada de cada

\footnotetext{
${ }^{20}$ Vid. "Protocolo de colaboración entre la Xunta de Galicia y la Comisión de Coordinación de la Región Norte de Portugal, para la realización de un estudio de viabilidad técnico-económico y de mercado de la conexión ferroviaria Oporto-Vigo $(20$ de julio de 199)". Disponible en URL: $<$ http://www.seap.minhap.gob.es/dms/es/web/areas/politica_autonomica/participacion-ccaaeu/Menu_Coop_TransfrCooperacion/Coop_Transfronteriza/parrafo/00/text_es_files/2013_04_Informe_web_cooperacion_transfronterizax/2014_04_Informe_web_cooperacion_transfronteriza_.pdf $>$. Consultada el 20 diciembre de 2014 .
} 
uno de los Estados miembros en el club de la Unión Europea, momento en el que se da paso a la sympoliteia institucional comunitaria.

Las lecciones que nos aporta la construcción de Europolis amplían el conocimiento sobre el desfase que se produce entre el crecimiento de la cooperación transfronteriza estatal y regional en Europa, y la más clásica (preexistente) de la interdependencia transfronteriza local comunitaria. En este sentido, es fácil concluir que en estos últimos años de Iniciativas Comunitarias como Interreg, lo estatalregional e institucional ha dominado el panorama de la cooperación transfronteriza.

Por todas estas razones, el caso de la RIET nos permite comprobar cómo se crea Europolis a partir de prácticas mixtas de isopoliteia y sympoliteia, a través de expresiones de interés, hermanamientos, declaraciones constitutivas, protocolos de colaboración entre actores diversos con fines comunes. De esta manera, redes de ciudades, urbes y villas, universidades, asociaciones de carácter empresarial y/o cultural, trabajan la diversidad, desde la flexibilidad adaptativa y el método de la cooperación reforzada, en función de la agenda de cada uno y de la agenda general de la RIET. A partir de estos argumentos se puede concluir que:

1. La RIET se ajusta a un modelo de Europolis en el que prima la cooperación transfronteriza reforzada, en la que intervienen un alto grado de diversidad y flexibilidad tanto en la configuración de los objetivos como en la negociación y puesta en marcha de los procesos para alcanzarlos. Diversidad y flexibilidad que permiten crear identidad y pertenencia común a una estructura de cooperación transfronteriza formal-informal, que está en línea con la agenda de cada uno de los actores de la red, y que permite, como en las ligas clásicas, mantener un alto nivel de autonomía institucional.

2. La RIET representa un modelo de cooperación que se mueve entre lo formal (cierto grado de institucionalidad jurídico-formal) e informal (prácticas abiertas de cooperación: Confederación de Empresarios, Asociaciones de Vecinos, Universidades, etc.), de carácter innovador y exponente de un tipo de cooperación de "segunda generación" que tiene espacio y legitimidad para la gestión de los asuntos públicos y comunes de los territorios de frontera.

3. La RIET es un actor clave, que ayuda a definir discurso transfronterizo y a negociar contenidos de la agenda pública para las Cumbres Ibéricas: en el 2013 no participa pero prepara discurso, mientras que en el 2014 participa en la fase previa y ayuda a marcar la agenda de 2015.

4. La RIET ayuda a conformar una "agenda sistémica" específica para los territorios de frontera de España y Portugal, a partir de su rol de lobby y estrategia de interlocución con los medios de comunicación lusos y españoles. Todo ello define una Europolis transfronteriza desde el discurso de la proximidad, mejorando su visibilidad ante el conjunto de decisores públicos estatales y comunitarios. 
5. Finalmente, la RIET refuerza la posición de los espacios locales (urbanos y rurales) como actores protagonistas de la cooperación transfronteriza en la agenda pública comunitaria; "ventana de oportunidad" que los actores subnacionales (también los regionales), podrían aprovechar impulsando nuevas institucionalidades del estilo de Europolis, basadas en la proximidad, la diversidad y la flexibilidad.

Tras el planteamiento del objetivo de la investigación y el proceso de análisis posterior, es posible confirmar que la creación de la RIET, y su inmediato desarro1lo, ha conseguido en breve espacio de tiempo (2009-2014), abrir el modelo de cooperación transfronteriza formal a otras organizaciones de carácter formalinformal, así como introducir en las agendas públicas estatales demandas locales desde procesos innovadores de selección de problemas y gestión de dichas agendas. El análisis de la evolución institucional de la RIET y de las agendas de las Cumbres Ibéricas de 2013 y 2014, para el caso de la frontera de España-Portugal, avanza respuestas a las preguntas concretas sobre problemas públicos transfronterizos, como es el caso de la movilidad; si bien es cierto, que el carácter exploratorio de la investigación y la novedad de la institución no permiten concluir una influencia decisiva en la configuración de la agenda pública estatal común de Portugal y España.

Los próximos años, centrados ya en el desarrollo de la agenda comunitaria 20142020, determinarán el potencial de este tipo de estructuras flexibles de cooperación formal-informal para el conjunto de la Cooperación Territorial Europea. Mientras tanto es preciso recordar que la construcción de una Europolis como modelo de cooperación de "segunda generación", precisa de la participación y legitimación de la sociedad civil, los ciudadanos y vecinos; actores sociales que aún deben ganar protagonismo en el espacio institucional, ayudando a recordar en qué consistían las buenas prácticas de la cooperación informal, antes de que las instituciones formales ocuparan todo el espacio posible de convivencia transfronteriza.

\section{Bibliografía}

Bauman, Zygmunt (2009) Tiempos líquidos. Vivir en una época de incertidumbre. Barcelona: Tusquets Editores.

Cancela, Celso; Cordal, Constantino; Domínguez, Luis, y Varela, Enrique José (2012) "The «formal» and «informal» cross-border cooperation in Europe", en I. Pires (comp.) Borders and Borderlands: Today's Challenges and Tomorrow's Prospects. Lisbon: Centro de Estudos Geográficos, 61-73.

Comisión Europea (2014) "Inversión para el empleo y el crecimiento. Promoción del desarrollo y la buena gobernanza en las regiones y ciudades de la UE. Sexto 
informe sobre la cohesión económica, social y territorial". Política Regional y Urbana. Bruselas: Comisión Europea.

De la Macorra y Cano, Luis Fernando (2011) La Eurociudad Elvas/Badajoz en el Suroeste ibérico y europeo. Badajoz: Imcrea.

Domínguez, Luis (2004) "Europa e a Fronteira Luso-Galaica: História e Reencontro", en L. Domínguez y N. Venade (coords.) As Euro-Regiões e o Futuro da Europa: O Modelo da Euro-Região Galiza-Norte de Portugal. Porto: Eixo Atlântico do Noroeste Peninsular, 3-51.

Domínguez, Luis, y Pires, Iva (eds.) (2014) Cross-Border Cooperation Structures in Europe. Learning from the Past, Looking to the Future. Bruxelles: Peter Lang. Eckstein, Harry (1975) "Case Study and Theory in Political Science", en F. Greenstein y N. Polsby (eds.) Handbook of Political Science. Reading, MA: AddisonWesley, 79-138.

Eixo Atlântico (2013) "La RIET elige al alcalde de Viana de Castelo como nuevo presidente". [Disponible en URL: $<$ http://www.eixoatlantico.com/?q=es/node/1718>. Consultado el 23 de setiembre de 2014].

Goldsmith, Michael (1994) "Europa y las Regiones: Europa y las Ciudades", en M. Arenilla, J. Loughlin y T. Toonen (eds.) La Europa de las Regiones. Una perspectiva intergubernamental. Granada: Universidad de Granada, 173-185.

Hocking, Brian (2004) "Patrullar por la «frontera»: la condición de actores de los gobiernos no centrales", en F. Morata (ed.) Gobernanza multinivel en la Unión Europea. Valencia: Tirant lo Blanch, 395-426.

Kohler-Koch, Beate, y Rittberger, Berthold (2006) “The «Governance Turn» in EU Studies". Journal of Common Market Studies, vol. 44 (Annual Review), 27-49.

Lois, María (2013) "Re-significando la frontera: El caso de la Eurociudad ChavesVerín”. Boletín de la Asociación de Geógrafos Españoles, núm. 61, 309-327.

Lois, María, y Cairo, Heriberto (2011) "Desfronterización y refronterización en la Península Ibérica". Geopolítica(s), vol. 2, núm. 1, 11-22.

Lois, María, y Cairo, Heriberto (2015) "Heritage-ized Places and Spatial Stories: B/Ordering practices at the Spanish-Portuguese Raya/Raia". Territory, Politics, Governance, vol. 3, núm. 3, 321-343.

Lois, María; Limón, Pedro, y Díaz, Marina (2015) "Las Eurociudades de la Raia: de Valença de Minho a Ayamonte. Experiencias de investigación". Comunicación presentada al I Congreso Internacional sobre Fronteras Comparadas (Universidade de Vigo), Pontevedra, 24 y 25 de septiembre de 2015, 1-17.

López, Álvaro X. (1998) A Galicia Irredenta. Vigo: Edicións Xerais.

Magnette, Paul (1999) La Citoyenneté européenne. Droits, politiques, institutions. Bruselas: Editions de l'Université de Bruxelles.

Magnette, Paul (2003) "European Governance and Civic Participation: Beyond Elitist Citizenship?". Political Studies, vol. 51, núm. 1, 144-160.

Magnette, Paul (2005) Citizenship: The History of an Idea. Londres: ECPR. 
Manheinn, Jarol B., y Rich, Richard C., (1988) Análisis político empírico. Métodos de investigación en ciencia política. Barcelona: Alianza Editorial.

Medina, Eusebio (2006a) "Aportaciones para una epistemología de los estudios sobre fronteras internacionales". Estudios Fronterizos, vol. 7, núm. 13, 9-27.

Medina, Eusebio (2006b) "Orígenes históricos y ambigüedad de la frontera hispano-lusa (La Raya)". Revista de Estudios Extremeños, vol. 62, núm. 2, 713-724.

Medina, Eusebio (2012) "La cooperación transfronteriza de «segunda generación» entre España y Portugal naturaleza, problemática, estrategias y perspectivas". Spanish Journal of Rural Development, vol. 3, núm. Extra 2 (Ejemplar dedicado a: Ponencias del Congreso Fronteras Ibéricas), 9-18.

Middelaar, Luuk van (2013) El paso hacia Europa. Madrid: Galaxia Gutenberg.

Morata, Francesc (2004) "Regiones y Gobernanza Multinivel en la Unión Europea", en F. Morata (ed.) Gobernanza Multinivel en la Unión Europea. Valencia: Tirant lo Blanch, 19-50.

Pascual, José (2007) "La sympoliteia griega en las épocas clásica y helenística". Gerión, vol. 25, núm. 1, 167-186.

Peña, Sergio (2007) "Cross-Border Planning at the U.S.-Mexico Border: An Institutional Approach". Journal of Borderlands Studies, vol. 22, núm. 1, 1-18.

RIET (2012) "Laguna de Duero reúne a la Comisión Ejecutiva de la Red Ibérica de Entidades Transfronterizas, RIET". [Disponible en URL: $<\mathrm{http}: / /$ www.rietiberica.eu/es/actualidad/noticias/item/136-laguna-de-dueroreune-a-la-comision-ejecutiva-de-la-red-iberica-de-entidades-transfronterizasriet>. Consultado el 23 de setiembre de 2014].

RIET (2014) "RIET impulsará la creación de una interparlamentaria diputados e eurodiputados de los territorios de la frontera ibérica". [Disponible en URL: $<\mathrm{http}: / / \mathrm{www}$.rietiberica.eu/es/actualidad/noticias/item/282-riet-impulsara-lacreacion-de-una-interparlamentaria-diputados-e-eurodiputados-de-losterritorios-de-la-frontera-iberica>. Consultada el 7 de abril de 2015].

Rojo, Argimiro, y Varela, Enrique J. (2011) "Las eurorregiones como motores de la refundación europea: una aproximación politológica”. Razón y Palabra, núm. 74, $1-17$.

Selltiz, Claire; Wrightsman, Lawrence S., y Cook, Stuart W. (1980) Métodos de investigación en las relaciones sociales. Madrid: Ediciones RIALP.

Torres, Chus (2010) "Quién, qué, cuándo, por qué y cómo. Comunicar el Eixo Atlántico". Eixo Atlântico. Revista da Eurorrexión Galicia-Norte de Portugal, núm. 17, 193-199.

Trillo-Santamaría, Juan-Manuel y Paül Valerià (2014) "The Oldest Boundary in Europe? A Critical Approach to the Spanish-Portuguese Border: The Raia Between Galicia and Portugal". Geopolitics, vol. 19, núm. 1, 161-181.

Steinmo, Sven (2013) "Institucionalismo histórico", en D. Della Porta y Michael Keating (eds.) Enfoques y metodologías de las ciencias sociales. Una perspectiva pluralista. Madrid: Ediciones Akal, pp. 131-151. 
Unión Europea (2011) Las ciudades del mañana. Retos, visiones y caminos a seguir. Bruselas: Comisión Europea, Dirección General de Política Regional.

Varela, Enrique J. (2010) "Gestión pública y gobernanza local en perspectiva comparada. Las políticas públicas de modernización administrativa en los gobiernos locales de Galicia y el Norte de Portugal". Madrid: Universidad Complutense de Madrid, Facultad de Ciencias Políticas y Sociología [Puesto en línea el 30 de septiembre de 2009. URL: <http://eprints.ucm.es/10715/1/T31557.pdf>. Consultado el 20 de diciembre de 2014].

Varela, Enrique J. (2013) "El ADN de la cooperación transfronteriza, La cooperación informal en la Eurorregión Galicia-Norte de Portugal", en R. Barajas y F. Aguilar (coord.) Interdependencia, cooperación, gobernanza y fronteras: ¿cuál es el eslabón perdido? Tijuana, Baja California: Colegio de la Frontera Norte, 329-356.

Varela, Enrique J. (2015) "Reseteando la gobernanza transfronteriza: Explorando nuevos «cleavages» cooperativos «formal-informal», «material-inmaterial»", en R. Barajas, N. Oddone y P. Wong (eds.) Fronteras y procesos de integración. Experiencias comparadas en regiones transfronterizas, perspectivas teóricas y evidencias empíricas. Tijuana, Baja California: Colegio de la Frontera de Norte, 49-69.

Varela, Enrique; Cancela, Celso, y Cordal, Constantino (2015) "The Consolidation of the Euro-regional Territory and Its Consequences: The Case of the GaliciaNorth of Portugal Euro-region". The Journal of Territorial and Maritime Studies, vol. 2, núm. 1, 77-95. 